\title{
¿Aprendemos botánica en contextos informales? Revisión teórica y narraciones de aprendizajes
}

\author{
Do we learn Botany in informal contexts? Theoretical review and learning narratives
}

Emiliano Foresto ${ }^{1}$

\section{Resumen}

Este ensayo ha abordado el concepto de aprendizaje informal, con énfasis en los aprendizajes botánicos que adquirimos fuera de los espacios formales, en el hogar, viajes, con amigos, el trabajo, etc. Cuando pensamos en botánica, creemos que es un conocimiento para unos pocos, y lo asociamos a los planes de estudio de las carreras de grado con orientación biológica; sin embargo, el aprendizaje sobre esta ciencia en nuestra vida cotidiana es más natural de lo que parece. Se realizó una pequeña indagación bibliográfica y conceptual sobre la terminología, y una recopilación de relatos sobre algunos tipos de aprendizajes vinculados a la botánica que las personas aprenden por diferentes medios y contextos. Como resultado se propone reflexionar la interdependencia entre los ámbitos de aprendizajes, para alejarnos de esa visión de compartimentos estancos, eliminando las etiquetas y pensando en la idea de aprendizajes integrados.

Palabras clave: Aprendizaje informal; botánica; contextos; aprendizaje integrado; ecología de aprendizaje.

\section{Abstract}

This essay has addressed the concept of informal learning, with an emphasis on botanical learnings that we acquire outside of formal spaces such as at home, traveling, with friends, work, etc. When we think of botany, we believe that it is knowledge for few people, and we associate it with the study plans of undergraduate courses with a biological orientation; however, learning about this science in our daily lives is more natural than it seems. A short bibliographic and conceptual inquiry was made on terminology, and a compilation of stories about some types of learning related to botany that people learn through different means and contexts. As a result, it is proposed to reflect on the interdependence between the areas of learning, to move away from that vision of watertight compartments, eliminating labels and thinking about the idea of integrated learning.

Keywords: Informal learning; Botany; contexts; integrated learning; learning ecology

\section{Introducción}

Este ensayo se enfoca en el aprendizaje informal, con énfasis en los aprendizajes botánicos que adquirimos fuera de los espacios de la educación formal.

En una oportunidad regresé de la Pampa con un brote de una planta que en aquella zona denominaban chilladora por el chasquido constante que producían cuando se prendían fuego. Es una chilladora sentenciaban los lugareños. Era un arbusto pinchudo, de hojas pequeñas y una flor bonita en los tonos del amarillo y el ocre. Fui a rectorado y pedí hablar con el rector-destacado botánico- a la secretaria. Volvió al momento informando que estaba en una reunión y que si me parecía le transmitiera el asunto de la consulta. Saqué el sobre con el brote y dije simplemente: Quisiera saber cómo se llama esta planta que le dicen chilladora. La secretaria quedó sorprendida por el tenor de la consulta, pero fue al lugar de reunión volviendo al rato con una sonrisa y un

\footnotetext{
1 Ingeniero Agrónomo. Docente de Botánica Sistemática Agrícola.. Departamento de Biología Molecular, Facultad de Ciencias Exactas, Físico-Químicas y Naturales. Departamento de Biología Agrícola. Facultad de Agronomía y Veterinaria. Universidad Nacional de Río Cuarto (UNRC), Río Cuarto, Córdoba, Argentina. eforesto@ayv.unrc.edu.ar https://orcid.org/0000-0002-8196-3030
}

Recibido: 14/10/2020 - Aprobado: 14/11/2020 
papel en el que se leía: Chuquiragua erinacea. Después descubrí que había muchas chuquiragas distintas, con morfología y flores particulares. En épocas de internet es más fácil buscar si se sabe qué se busca o si se anima a dar rienda suelta a la curiosidad (Donolo, 2019: 229).

Desde algunos enfoques culturales y socio constructivos de la psicología el aprendizaje puede ser comprendido como un proceso social, situado y distribuido. Es un proceso porque demanda de su tiempo y se da a lo largo de toda la vida. Es social porque aprendemos en interacción con otros, nuestras habilidades intelectuales, sociales y emocionales se conforman producto de la vinculación con diversas personas en una variedad de ambientes. Es situado, debido a que la práctica de aprender se desarrolla en un espacio y un tiempo, dando por resultado configuraciones particulares en las que confluyen lugares, objetos, recursos, personas y conocimientos, entre otros. Es distribuido entre las personas y los contextos permitiendo al aprendiz acceder a una mayor variedad de recursos que le permiten construir conocimientos sin necesidad de estar dentro del aula únicamente (Melgar y Donolo, 2011).

Los diferentes saberes que se pueden aprender incluyen conocimientos, y otros tantos más como: practicas, destrezas, habilidades, mitos, creencias, tradiciones, ritos y valores que los seres humanos aprenden para darle sentido a la vida desde aspectos sociales, culturales, económicos, etc. El proceso de aprendizaje se desarrolla desde temprana edad en diversos contextos ecológicos y socioculturales. Como propone Dewey (1896), "desde los primeros años de vida dependemos de otros, los observamos, nos comunicamos y aprendemos de ellos (a través de procesos como la imitación), modelando así nuestra percepción y comprensión del ambiente". Es por eso que los diferentes contextos, ámbitos o espacios en donde se desarrollan estos procesos juegan un papel fundamental en el aprendizaje.

Con esta perspectiva más amplia del aprendizaje se puede advertir que convivimos con una amplia variedad de contextos de aprendizaje que no se reducen solamente a los espacios formales. Foresto (2020a) plantea la existencia de diferentes aprendizajes en función del contexto o ámbito donde se desarrollan: formales, no formales e informales. El aprendizaje formal incluye a la educación escolar, más precisamente a los aprendizajes que se suscitan en el sistema escolar, que se encuentran organizados en un sistema educativo, altamente institucionalizado, cronológicamente graduado y jerárquicamente estructurado que se extiende desde los primeros años de la escuela primaria hasta los últimos años de la universidad, pudiendo las personas vivenciar todas las instancias o sólo alguna de ellas (Trilla, Gos, López y Martin, 2003; Aguirre Pérez y Vázquez Molini, 2004). El aprendizaje no formal, es intencional, la persona que asiste a estas formas de educación lo hace por razones propias, y los programas se organizan para el aprendizaje, llegando a complementar, apoyar o como una fuente de valorización del aprendizaje de las experiencias adquiridas formalmente (Melnick y Botez, 2014). Por último, es un proceso que dura toda la vida y en el que las personas adquieren y acumulan conocimientos, habilidades, actitudes y modos de discernimiento mediante experiencias diarias y su relación con el medio social, cultural, ambiental, económico y político del que participan (Trilla, 1988). Este concepto de aprendizaje a lo largo de la vida se basa en la idea de aprendizaje permanente, donde las personas tienen la capacidad para aprender siempre, en diferentes ámbitos y en diferentes momentos de su vida. Se aprende a "lo ancho" y a "lo largo" de la vida, debido a que los procesos de aprendizaje juegan un papel central en el desarrollo del ser humano.

Cuando pensamos en la "botánica", la imagen que nos viene inmediatamente a la cabeza es un jardín lleno de plantas. Sin embargo, ofrecer una definición precisa de esta disciplina resulta algo más complicado. Según el diccionario, la botánica es la "ciencia que estudia la estructura, las características, las propiedades y las relaciones de los vegetales y sus procesos vitales." Es bien sabido que la botánica forma parte de la currícula obligatoria en estudios secundarios con orientaciones en ciencias de la vida y algunas carreras universitarias (Ingeniería Agronómica, Profesorado y Licenciatura en Biología, Ingeniería Forestal, Farmacia, Ciencias del Mar, entre otras). Algunas de las preguntas que nos planteamos e interpelan son: ¿Sólo aprendemos botánicas en contextos formales? ¿qué "morfologías" tienen los aprendizajes?, ¿aprendemos botánica en contextos informales?, ¿qué aprendizajes botánicos se aprenden inter-existiendo con otros?, ¿cuáles son las primeras aproximaciones con la botánica que desarrollan las personas?, ¿será que aprender en la "naturaleza" es más "natural" de lo que parece? 
Este ensayo realiza una revisión exploratoria sobre los aprendizajes informales para mostrar los avances en el campo de estudio con un fuerte impacto en la investigación educativa, a continuación, se realiza una recopilación de relatos sobre las formas que va tomando los aprendizajes botánicos, que son vivenciados por diferentes personas en diferentes contextos de su vida cotidiana.

\section{Desarrollo}

\section{Aprendizajes informales: Perspectivas teóricas}

El aprender es un proceso amplio, integral y continuo en el cual los sujetos desarrollan o adquieren conocimientos, habilidades y actitudes para sobrevivir y responder creativamente a los cambios en el medio, evolucionar, transformar y progresar (Corredor, 2003). El aprendizaje informal se identifica como "el proceso de toda la vida mediante el cual cada persona adquiere y acumula conocimientos, habilidades, actitudes e ideas de las experiencias diarias y la exposición al medio ambiente" (Coombs y Ahmed, 1974, p. 43). Los aprendizajes informales se presentan en diversidad de ambientes indiferenciados y subordinados a otros procesos sociales; inmersos en otras realidades culturales (Trilla, Gros, López y Martin, 2003), sin vinculación aparente con las formas canónicas tradicionales de los contextos formales de aprendizaje.

Foresto da un concepto holístico afirmando que: "El aprendizaje informal nos acompaña toda la vida, desde el nacimiento hasta la muerte las personas siguen aprendiendo. Y por eso decíamos que se asocia al Aprendizaje a lo Largo de la Vida. Algunos de los aprendizajes más importantes los hacemos de manera informal, en la familia, en la comunidad, en el sistema escolar, en el trabajo, en el deporte, conversando, leyendo y escribiendo, debatiendo, en contacto con la naturaleza, con los medios de comunicación, con las artes, con internet, etc., vamos aprendiendo múltiples saberes, prácticas y habilidades en el transcurrir de la vida, a menudo, las personas no son conscientes de lo que aprenden, sin habérselo propuesto como objetivo. En cierta forma el Aprendizaje a lo Largo de la Vida integra los tres tipos de aprendizaje: formal, no-formal e informal. Cada persona tiene su propia combinación y su propia trayectoria de aprendizaje que es única y especifica. Algunas en su vida tienen mucho aprendizaje formal y no-formal, otras tienen poco o ningún aprendizaje de este tipo. Todos en cierta forma desarrollamos aprendizajes informales, aprendizajes que son indispensables para la vida, para la convivencia, para el trabajo y para el cuidado del medioambiente (Prácticas agroecológicas, de permacultura, entre otras; Foresto, 2020, p.16).

En los contextos informales las personas pueden desarrollar conciencia, habilidades, interés, motivación, competencias sociales y prácticas, e identidades en su trayectoria de aprendizaje (Martin y Donolo, 2019). El conjunto experiencias, capacidades, situaciones sociales y nuevas oportunidades para aprender continúan a lo largo de la vida de una persona (Bell, Lewenstein, Shouse y Feder, 2009). Otros autores (Alves da Silva y Ferreira, 2016) sostienen que el "aprendizaje informal es el proceso por el cual las personas adquieren los conocimientos, habilidades y actitudes con la experiencia cotidiana y la exposición al medio ambiente en el que viven" (2016:8)

El aprendizaje es un proceso y a la vez resultado, es una práctica individual y una construcción colectiva que se da en un contexto, en una realidad multidimensional. “¿Qué conocimiento se adquiere y por qué?, ¿dónde, ¿cuándo y cómo se utilizan?, constituyen preguntas esenciales tanto para el desarrollo de los individuos como de las sociedades" (UNESCO: 2015, p.17). El informe de la UNESCO denominado "Aprender a ser: el nuevo mundo de la educación de hoy y de mañana" a cargo de una comisión internacional donde el responsable es Pierre Faure (1972), un pionero en el campo del Aprendizaje a lo Largo de la Vida (ALV) , en las cuales se destacan algunas valiosas ideas: "el amor por el aprendizaje crea un deseo de aprendizaje a lo largo de la vida" "la meta del ALV es lograr empoderar a las personas para que tomen el control del desarrollo económico, científico y tecnológico de una manera democrática". 
Si aprender es el asunto de toda una vida, en su duración y en su diversidad, y de toda una sociedad, tanto en lo que concierne a sus recursos educativos como a sus recursos sociales y económicos, entonces es preciso ir más allá de la necesaria revisión de los "sistemas educativos" y pensar en el plano de una ciudad educativa".

En este sentido Livingstone (1999 en Schugurensky, 200o) considera que, el aprendizaje informal puede definirse como una actividad que involucra la búsqueda de comprensión, conocimiento o habilidades que ocurren fuera del currículo formal y no formal; en este sentido resulta interesante rescatar un fragmento de Schugurensky (2000):

Es importante tener en cuenta que estamos utilizando deliberadamente la palabra "aprendizaje" y no "educación", porque en los procesos de aprendizaje informal no hay instituciones educativas, instructores institucionalmente autorizados o currículos prescritos. También es pertinente tener en cuenta que estamos diciendo 'fuera de los planes de estudio de las instituciones educativas' y no 'fuera de instituciones educativas', porque el aprendizaje informal también puede tener lugar dentro de instituciones educativas no formales. En ese caso, sin embargo, los aprendizajes ocurren independientemente (y en ocasiones en contra) de los objetivos previstos del plan de estudios explícito (Schugurensky, 2000:2).

Considerando los ambientes de aprendizaje Illera (2018), enfatiza en el interés creciente por entender bien los contextos (lugares, sitios, escenarios) en los que se produce el aprendizaje, no sólo por los contextos institucionales, sino sobre todo, por aquellos otros que se dan en otros ámbitos sociales como la familia, el trabajo, las amistades o, en general, la «vida cotidiana» (Trilla, 1986; Hager, 2012; Livingstone, 2006; Vadeboncoeur, 2006). Este interés ha hecho surgir la idea de que es necesario comprender no solo los aprendizajes que se producen sino también su interrelación, lo que a veces se denomina su ecología, o integración, sus dependencias mutuas (Coll, 2013). Más focalizado en los procesos cognitivos que en los ambientes de aprendizaje, Schugurensky (2000), propone tres formas de aprendizaje informal utilizando criterios como la intencionalidad y la conciencia: Autodirigido (cuando consiste en un aprendizaje consciente e intencional, que se desarrolla de manera individual o como parte de un grupo, sin la ayuda de un facilitador o guía), Incidental (es un aprendizaje no intencional, pero la persona es consciente de la experiencia que se produce cuando él no tiene ninguna intención previa de aprender algo) y Socialización (es inconsciente y no intencional, se lleva a cabo cuando las personas interiorizan nuevas actitudes, habilidades, comportamientos, etc.). A pesar de su interés, las subdivisiones entre formas del aprendizaje informal son difíciles por ser poco claras, en este sentido el aprendizaje informal parece más una construcción teórica externa, de la que el propio sujeto puede no ser consciente en algunos casos, como cuando acontece incidentalmente o en determinadas formas de su vida diaria (Rodríguez Illera y Kaechele, 2009).

La definición de aprendizaje informal está sesgada por aquellos esfuerzos por diferenciar o comparar el aprendizaje informal y formal, y estas opiniones dicotomizadas con frecuencia son simplificaciones excesivas de las características del aprendizaje informal. Sin embargo, la naturaleza del aprendizaje en ambientes informales es mucho más compleja. Debido a que tal aprendizaje es complejo, el intento de dicotomizar es un intento de reducir la complejidad inherente a la naturaleza intrincada del aprendizaje y la diversidad de puntos de vista y valores (Anderson y Ellenbogen, 2012). Illera (2018) en esta línea de pensamiento refiere a que, los discursos psicológicos y pedagógicos han insistido en el proceso de aprendizaje como explicativo de sus resultados, más precisamente como resultados escolares. Así variados estudios pusieron su mirada en el conocimiento escolar, centrándose fundamentalmente en aspectos "cognitivos", asociados con razonamientos formales o abstractos que se conectan con otras áreas del conocimiento escolar, con un carácter de tipo conceptual. Estos saberes se van tejiendo con otros más "lejanos" o "complejos" los cuales tienen que ser aprendidos en grados superiores. Generalmente, aunque hay excepciones, el conocimiento que se suscita "fuera de la escuela" generalmente es poco atendido o valorado por los escolares. Estos tipos de aprendizaje son más fragmentados y desconectados, la segmentación refiere a que lo aprendido en un determinado contexto está separado de lo que se aprende en otro diferente (Moss, 2001). La educación formal y sus variantes (currículum explícito, agentes profesionales para enseñarlo, evaluación y sanción 
social de lo aprendido), se contrapone con lo que ocurre en situaciones de la vida cotidiana -que solo son «informales» cuando las comparamos con la escuela- se basa en mecanismos de aprendizaje básicos y casi siempre "espontáneos": imitación-mímesis, juego, soporte mediante andamiaje, participación progresiva en el grupo, casualidad, narrativa y casos, comunidades de práctica, etc.

El aprendizaje informal puede ser pensado de muchas maneras, algunos contextos tan diferentes como el autoaprendizaje, aprendizaje dirigido o instrucción, que son contextos directamente organizados para "aprender" como objetivo último, dirigido conscientemente por el propio aprendiz que auto regula su aprendizaje, objetivos y ritmo (aprendizaje explícito). Por otro lado, los acontecimientos casuales, que son incidentales o espontáneos, donde no hay intención de aprender en sí, simplemente se aprende por el hecho de "estar ahí" o "de estar en el tiempo y espacio justo" en donde se adquiere el aprendizaje como algo claramente social y cultural (Collins, 2010). A este tipo de aprendizaje informal se lo denomina aprendizaje implícito, se desarrolla de manera automática y no intencional, de manera no consciente, mediante asociaciones principalmente, en situaciones cualesquiera y, en muchos casos, sin intervención de la voluntad, la atención o la concentración. El aprendizaje implícito sucede, aunque no con un fin, se determina por el sensorium humano, y se sucede con el solo hecho de estar presente en un contexto o situación y recibir sus estímulos. Además, existe contextos híbridos o Ecologías de Aprendizaje (trabajo, comunidades y vida cotidiana), Jackson (2013, p.7) sugiere que "las Ecologías de Aprendizaje comprenden los procesos y variedad de contextos e interacciones que concede al individuo las oportunidades y los recursos para aprender, para su desarrollo y para alcanzar sus logros". Así algunos ámbitos de aprendizaje se presentan unidos de manera "transparente", sin costura, como si estuvieran profundamente coordinados. En este sentido, Rodríguez Illera (2015) refirió a los contextos de actividad y aprendizaje a través de características comunes a todos ellos, aunque muy generales: los marcadores de contexto que nos permiten diferencia en presencia de qué contexto o actividad estamos, un foco que incluye la parte visible de la actividad que se lleva a cabo (p. e. aprender, jugar, pasear, leer, conversar, hacer deporte, etc.), y también un trasfondo que es el conjunto de supuestos (personales, culturales, históricos, sociales ) sobre los que se organiza la actividad.

Los aprendizajes no son únicos, ya que se van construyendo a lo largo de la vida de las personas en diferentes entornos. Como recuerda Falk (2002, p.2) "no hay una única forma de aprender, ni un sólo lugar o momento en el que aprendamos. Todo aprendizaje tiene un lugar continuo, de muchas fuentes y de muchas maneras diferentes".

\section{Aprendizajes botánicos en contextos informales ¿Es posible?: Relatos de aprendizajes}

En revisión de trabajos previos que versen sobre las formas, los contextos y tipos de aprendizajes informales, encontramos que aparecen más documentados en la bibliografía los que refieren a ciencia y TIC (Bell, Lewenstein, Shouse y Feder, 2009; Matamala Riquelme, 2016; Ibáñez Etxeberria, Vicent Otaño y Brouard, 2012; Fontanillas, Catasús, Raffaghelli, y Morer, 2020), en este sentido los trabajos se abocan aprendizaje informal con dispositivos móviles, en museos virtuales, con trabajos de laboratorios, relativos a patrimonio, internet, redes sociales, entre otros. Muchas investigaciones también apuntan a el Trabajo (Adobe, 2007; Lin y Bound, 2011) en donde las personas aprenden de forma espontánea e improvisadas, y esas prácticas de aprendizaje que, en algunos casos se retroalimentan en el trabajo colaborativo (Martin y Donolo, 2019). Mientras que otras en menos medida se encargan de estudiar Artes (Huerta y Alonso-Sanz, 2017), practicas agroecológicas (Gasull, Herrador y Martin, 2020), relaciones sociales y habilidades para la vida (Matamala Riquelme, 2016).

Si bien en la literatura se realizan estudios que se encargan de atender a los contextos informales de aprendizajes y las formas que este puede tomar, son nulos los estudios que atienden a las formas de aprendizajes informales en relación a saberes botánicos. Aparecen muchos escritos que abordan el aprendizaje botánico desde diferentes perspectivas, formas y variantes, pero siempre inmersos en contextos formales de aprendizaje (Santos y Tellez, 2011; Salgado y Guevara, 1994; Moreno, 2007). La botánica en gran parte sólo es pensada desde un punto netamente biológico, sin embargo, está directamente relacionada con la sociedad, es fundamental para la vida del hombre, ya que interviene en situaciones y objetos que forman 
parte de la vida diaria, todo lo que comemos directa o indirectamente, los objetos de decoración de nuestro hogar, los muebles, nuestra vestimenta, las hojas de papel en donde escribimos, nuestras costumbres, etc. Es por esto que es importante recuperar esos aprendizajes informales para poder comprender mejor nuestro ambiente y aprender a convivir con el sin dañarlo. Estamos en la sociedad del conocimiento y la información que genera alienta un tipo particular de cultura del aprendizaje. El conocimiento parece ser cada vez más inabarcable, aproximarse a comprender y aprender "de otro modo" sobre el saber (Adúriz Bravo, 2005), es un gran desafío que se presenta para las instituciones de Educación, donde se vislumbra la posibilidad de aprender inter-existiendo con nuestra tierra y recuperando saberes previos. Ausubel (2002) afirma:" Si tuviera que reducir toda la Psicología Educativa a un solo principio enunciaría este: El factor más importante que influye en el aprendizaje es lo que el alumno ya sabe."

Para recuperar algunas experiencias de los aprendizajes botánicos más significativos de las personas en su vida cotidiana elegimos iniciar las conversaciones con 15 personas, de forma exploratoria mediante Whatsapp, por medio de mensajería de texto, audios y en algunos casos realizamos sesiones de videollamada, lo que nos permitió conocer de una manera más cercan e íntima el contexto de estudio (Martin y Donolo, 2019). El sistema de mensajería de WhatsApp (aplicación de mensajería para teléfonos inteligentes, que envía y recibe mensajes mediante Internet https://es.wikipedia.org/wiki/WhatsApp.) es un tipo de lenguaje virtual instantáneo, que completa los servicios de correo electrónico, de mensajes cortos o sistema de mensajería multimedia. Es un sistema que comparte algunas peculiaridades de otras técnicas de comunicación electrónicas pero que aporta adicionalmente elementos novedosos como el código iconográfico emoji (Martin y Donolo, 2019; Calero Vaquera, 2014). Esto permitió realizar entrevistas más distendidas con cierta amplitud en la confección de preguntas, además los entrevistados contaban con más tiempo para pensar lo que se quiere decir, asi se consiguen respuestas abiertas para que los entrevistados puedan explayarse al comunicar su experiencia de aprendizaje libremente (Vicario, Chiecher, Amieva, Fernández y Ortiz, 2015).

Para hablar de aprendizajes botánicos en relación al Trabajo y TIC e Internet creemos que Sheila (Dueña de un salón de Tatuajes - 23 años) y Pablo (Jardinero - 35 años) pueden ser un buen ejemplo.

(..) soy bastante tecnológico, cuando se ir a los Jardines uso mucho mi celular, conozco muchas plantas por el hecho de que hace años que uno está en contacto. Me fue enseñando mi papa, y fui aprendiendo con la "vida". Pero como te decía, uso mucho el celu, cuando hay una planta que no conozco le saco una foto y luego uso una aplicación que se llama PlantNet, entonces vos subís la foto seleccionas si le sacaste la foto a una hoja, flor, fruto, corteza, etc; y la aplicación por una base de datos que tiene la procesa y te da el nombre de la especie el "posta" creo que le dicen científico, de ahí con el nombre me voy a google y la "googleo" para ver si es de sombra o sol, cuánta agua requiere, si se enferma y de más cuestiones que me interesen; ahora en estos tiempos que corren no basta con saber podar tenés que ser un jardinero 3.0" (Pablo).

Tatuar es mi pasión, al principio hacia cosas simples, pero sentí que mi trabajo necesitaba pegar un salto cuántico, así empezó a atraparme el mundo de los tatuajes botánicos, soy una amante de la naturaleza y estos tatuajes son más frescos, naturales y delicados, rompen todas las ideas preestablecidas de los tatuajes comunes. En este tiempo aprendí mucho de plantas, empecé a estudiar biología y deje en el $2^{\circ}$ año, pero uno de los profes nos había nombrado un sitio de internet Flora Argentina, ahí encentras muchas fotos y descripciones de la planta, cada vez que tengo que hacer un tatuaje de una planta, la busco en el sitio y aprendo de ella para conocer sus rasgos, estructuras, partes para poder dibujarla a la perfección sin perder detalle (Sheila).

Otro aprendizaje botánico informal muy interesante es el que aparece vinculado a las Artes, en este sentido Ivanna (Artesana de Vitrofusión - 50 años) nos versa como los saberes relacionados a las plantas que aprendió fueron necesarios para implementarlos en sus trabajos con vidrio. 
Soy maestra de nivel primario, pero siempre me gustó el Arte hace algunos años empecé a incursionar en la pintura, la joyería, el mosaiquismo y otras tantas más, hasta que empecé a tomar clases de Vitrofusión, con un grupo de mujeres y eso robo mi corazón. Siempre me gustaron las plantas, pero ahora mis piezas de vidrio tienen un toque botánico, ya que realizo algunos platos con de formas de hojas y flores. Recientemente realice un curso para incrustar hojas verdaderas en las piezas de vidrio que realizo, es por eso que me dedico a recolectar especies de plantas, teniendo en cuenta el tipo de flor que tienen, y las hojas, si son hojas muy blanditas no me sirven, los pinos por ejemplo aprendí que tienen hojas en forma de aguja, le dicen "aciculares", la cuestión es que cuando se secan se caen asique para mis trabajos no las prefiero. En estos aprendizajes fueron importantísimos mi abuela, mi hijo que es profesor de Botanica Agrícola y el internet (googleo mucho) (Ivanna).

En el relato se evidencia como su paso por las artes va hibridando desde contextos no formales (taller de Vitrofusión), luego informalmente ella "probando" hacer piezas, incrustando hojas verdaderas en sus trabajos debiendo aprender ciertos "caracteres botánicos" de las plantas que le son necesarios para su objetivo. Aquí ella rescata lo aprendido mediante su familia (los saberes de su abuela adquiridos informalmente y los de su hijo en espacios de formación universitaria) y el internet. El aprendizaje del arte generalmente se transita comenzando desde la no formalidad o informalidad, pero luego termina siendo un aprendizaje que se realiza para toda la vida.

La familia es sin duda uno de los entornos sociales donde se suscitan la mayor cantidad de aprendizajes, ya que opera en todo tiempo y lugar como el mejor instrumento para la trasmisión de tradiciones, creencias, convicciones y demás que se imprimen en quienes las reciben desde temprana edad. El contexto familiar es más descontracturado que el escolar, aquí intervienen gestos, risas, afectos, humores compartidos, experiencias. Las lecciones no están objetivamente estructuradas, el aprendizaje es implícito, a través del ejemplo y la imitación. Además, carece de una metodología específica, es más una respuesta a la demanda de los agentes sociales que intervienen. Para ilustrar este aprendizaje creemos que el relato de Nancy (Ama de casa - 70 años) puede servir para ejemplificar lo visto:

Mucho de lo que aprendí lo se gracias a mi familia, a mi madre le encantaba acomodar el patio, las plantas, las flores, tenía quinta también, ella le sabia el nombre a todas las plantas, sabia en que época florecían, cuales eran más susceptibles a las heladas, siempre mi madre nos decía que la malva por ejemplo la teníamos que usar para la cistitis o que la ruda era para limpiar la casa de las malas energías, siempre supe distinguir el olivo, porque para el domingo de ramos llevábamos ramitas para que el cura la bendijera (...). También recuerdo que teníamos una higuera en el patio, mi mama nos decía que las hojas no había que tocarlas, ella lo había aprendido por experimentarlo en su vida ya que cuando pasaba a juntar los huevos en el gallinero (donde había muchas higueras) e iba esquivando las ramas y luego le aparecían estas manchas rojas en la piel, años después yo leyendo una revista me entero que efectivamente es así producto de la reacción del sol con algo alergógeno (así decía en la nota) que tiene la planta, ahí comprobé que mi mama sin haberlo leído nunca lo aprendió y también nos transfirió ese conocimiento (Nancy).

En este relato también se aprecian conocimientos etnobotánicos que brindan alguna aproximación ente las relaciones entre plantas y personas y nos muestra la amplitud de usos que son trasmitidos de generación en generación (técnicas de limpieza energética, uso medicinal, plantas de importancia en cultos religiosos, efectos perjudiciales (alergias), entre otros). Siguiendo esta línea el relato de Jesica (Terapeuta holística y Artesana - 32 años) puede aportar más acerca de estos saberes en relación a la aromaterapia, en el mismo se advierte que ella hibrida entre aprendizajes informales vinculados a las plantas que adquiere a lo largo de su vida por múltiples vías, su primer contacto con estos saberes empiezan en un sus clases de yoga, pero también resignifica lo aprendido en su vida cotidiana, con sus amistades, su familia, en su trabajo, las películas románticas y por medio de internet. En palabras de la entrevistada: 
La aromaterapia siempre me atrapo, pero siempre lo relacionaba con tener que adquirir unos aceites esenciales que eran carísimos. Un día recuerdo fui a una clase de Yoga y mi profe nos sahumó antes de finalizar, con unos sahumos que tenían un aroma espectacular, me gustó tanto que ella me regalo uno, cuando llegue a casa empecé a averiguar, recuerdo que los desarme para saber con qué plantas estaban hechos, vi lavanda (a esa la conocía bien por mi abuela, ya que la tenía en su patio), laurel ( Infaltable en las salsas de mi mama los domingos con los tallarines) y pétalos de rosas ( esa la conocía, quien no vio una rosa, es el cliché clásico del amor de las películas).Con el tiempo empecé a buscar en internet y descubrí que también podía usarse Tuya, Salvia, Mirra y Enebro, entre otro (...). La historia finaliza en que me dedico a hacer y vender sahumos y yo misma gracias a mis aprendizajes en plantitas puedo ser capaz de recolectarlas e identificarlas (Jesica).

En cuanto a los aprendizajes relacionados a las practicas agroecológicas, las huertas y la cocina saludable, pensamos que el aprendizaje de Romina (Agente de seguros, vegetariana - 40 años) muestra como sin pensarlo un cambio en sus hábitos de vida género que de manera imprevista y espontanea vaya adquiriendo un montón de aprendizajes verdes.

A decir verdad estoy muy relacionada con todo lo verde soy vegetariana desde hace 6 años (...); el tener que cocinarme constantemente hizo que decida hacer una huerta en casa, al principio pensé que no sería posible, pero un amigo me ayudo a hacer la quinta en mi patio, removimos la tierra y pedimos las semillas de invierno a el INTA y a fines de abril el me ayudo a sembrarlas, me acuerdo que hacíamos surcos y tirábamos las semillas, jamás le hice cartelitos ni nada, cuando empezaron a nacer no tenía ni idea, asique no me quedo otra que aprender, buscaba fotos de la especie y intentaba sacarlas, fue fácil con el rabanito ya que crecía bastante y por la tierra asomaba su prominente raíz de color morado que es lo que se consume, el garbanzo formaba chauchas gorditas, la lechuga a esa la conocía y así las voy identificando para cosecharlas. Si alguna se complica más le mando fotos a mi amigo que es Ingeniero Agrónomo y me da una mano (Romina).

El último ejemplo nos resultó interesante, ya que en el caso de Martina (Estudiante de Abogacia - 19 años), mientras su mamá siendo docente universitaria dictaba un clase para alumnos de agronomía, de forma inesperada se encontró vivenciando un aprendizaje que era de carácter informal ya que fue repentino y accidental, pero por otro lado para los estudiantes que al mismo tiempo estaban del otro lado de la pantalla vivenciando una clase en un contexto universitario, correspondía a un aprendizaje formal. En este relato su mama en el rol de docente incentiva a sus estudiantes a que recuperen los aprendizajes informales y los puedan entrelazar con los conocimientos adquirido en el seno de los ámbitos formales.

Mi mamá es Ingeniera Agrónoma y mientras está dando sus clases por las plataformas meet o similar por la pandemia y la virtualidad yo aprendí bastante, recuerdo un día mi mama estaba dando clases con la esponja vegetal al lado de la compu, no entendía mucho, en un momento la agarra y les dijo a los alumnos ustedes saben que esto, (yo pensaba y si claro que lo van a saber "una esponja"), lo mismo responden los alumnos, mi mama pregunta: ¿de dónde sale la esponja?, en ese momento me di cuenta que nunca me lo había preguntado tampoco. Entonces ante el silencio ella continua: " $¿ S a b e n$ que la esponja es un zapallo?, que produce fibras que se utilizan en la industria, su género es Luffa y pertenece a la familia Cucurbitaceas”, ese día aprendí que la esponja sale de una planta y que es un tipo de zapallo." (Martina)

Para finalizar, un relato que permite apreciar como los aprendizajes informales vividos en la infancia con la familia o en nuestra vida cotidiana deben ser recuperados en los posteriores aprendizajes formales que se suscitan en las formaciones posteriores institucionalizadas, pensar que los estudiantes conocen lo que hemos enseñado y desconocen aquello que no se ha enseñado en las aulas es una idea muy generalista, que no da lugar a la posibilidad de poder aprender sobre la base de lo que ya conocen y re-significar aprendizajes previos. 
En patio de la casa de mi bisabuela Mafalda había una pérgola la cual estaba cubierta por una enredadera frondosa con flores vistosas en racimos grandes de color violeta, ella se sentaba ahí afuera en una mesa de cemento que había debajo de la pérgola, con su delantal azul, el mate dulce acompañándola y una bolsa grande llenas de "chauchas verdes" como ella les decía. Esa mañana yo me encontraba en su casa jugando mientras mama trabajaba, mi bisabuela estaba justo en ese lugar, sentada debajo de la enredadera, no pude contener mi curiosidad: “¿Qué es eso? ¿Por qué las cortas a ambos lados?" pregunte, a lo cual ella respondió, "son chauchas, son ricas, sabrosas llenas de vitaminas, adentro tienen semillas, les corto el cabito de adelante y el cabito de atrás de atrás antes de hervirlas porque eso no es tiernito para comerlo." Sin bastarme su respuesta exclame inocentemente desde adentro: “¿Qué es una semillita?” Ella respondió inmediatamente y sin dudar, "es la hijita de donde viene la planta de chaucha, de esa semillita puede nacer otra plantita que puede producir más chauchitas que luego nosotros comemos.” ¡Ahhhhh exclame! ¡Ahora si lo entiendo todo! Una década después cuando comenzaba mi primer año de la carrera de Ingeniería Agronómica me enteraría que el nombre de la hermosa enredadera de flores violetas era glicina (Wisteria sinensis (Sims) DC.)) y la famosa "chaucha verde" como la nombraba mi bisabuela era Phaseolus vulgaris L., las cuales eran especies que pertenecía a la familia Fabaceae, una familia cosmopolita con aproximadamente 19.500 especies diferentes, siendo la tercera familia de plantas más diversa del mundo (Foresto, 2020b:2)

\section{Conclusiones}

El aprendizaje no ocurre sólo en el aula, sino también en el hogar, el trabajo, en el lugar de juego, en la biblioteca, el museo, el parque; navegando por internet y en las interacciones cotidianas con otros. Cuando pensamos en botánica, imaginamos algo formal o pensado para "unos pocos", la propia denominación de "formal" nos recuerda algo serio y bien hecho, a sensatez, juicio, prudencia, mientras que "informal" lo pensamos como un sinónimo de inconstante y lo relacionamos a juerguista, poco cumplidor y poco comprometido, el lenguaje habla por sí solo y está cargado de simbolismos. Por ello, quizás, el aprendizaje informal ha sido siempre considerado en segundo lugar, por detrás del formal e incluso del no formal que, dentro de los aprendizajes definidos en ámbitos estructurados y sistematizados, son los que las personas eligen por su propia voluntad.

Desde tiempos remotos el hombre ha buscado en la naturaleza productos que puedan satisfacer sus necesidades básicas, las plantas son indispensables en la alimentación, la vestimenta, herboristería, fitoterapia, entre otros; lo cierto es que a lo largo de nuestra vida adquirimos una gran cantidad de saberes botánicos que seguramente desconocemos, porque de seguro no tomamos conciencia que seguimos aprendiendo a lo largo de nuestra vida. Esto nos muestra la frontera entre el aprendizaje formal e informal se diluye rápidamente, mucho de esto producto del cambio social. Lo que antes era un muro solido e inquebrantable, ahora es una franja más delgada, débil, permeable y osmótica, ya que es una puerta de entrada y salida de saberes, habilidades, destrezas, de comunicación de conocimiento, de relación constructiva; de los aprendizajes entre contextos formales e informales, que naturalmente están más hermanados.

En el desarrollo de los relatos ee puede observar cómo las personas a lo largo de su vida adquirieren saberes relacionados a las plantas a través de aprendizajes espontáneos o intencionales por medios informales que han sido el motor posterior de nuevas motivaciones, para sus trabajos, formas de vida, relaciones personales, actividades artísticas, etc. Esto nos invita a reflexionar los diferentes contextos donde aprendemos diversos saberes y replantearnos que este puede ser el inicio de empezar a transitar un camino de mayor interdependencia entre los ámbitos de aprendizajes, para alejarnos de esa visión de compartimentos estancos, eliminando las etiquetas y pensando en la idea de aprendizajes integrados.

A modo de conclusión, algunos interrogantes que nos interpelan, ¿podría ser el aprendizaje informal un elemento que sirve de andamio a la educación formal de grado y posgrado, como así también en la educación secundaria? ¿será necesario ampliar la visión y pensar en noveles ambientes que nos permitan profundizar en las diferentes ecologías de aprendizaje? ¿cómo empezar a diagramar estrategias de 
aprendizaje que aprovechen e interrelacionen el aprendizaje que se lleva a cabo en ambientes diversos potenciando los elementos claves que son esenciales en los procesos de aprendizaje? ¿qué valor les asigna la educación formal a los aprendizajes que se co-contruyen en los hogares, internet, amigos, trabajo?. Un enfoque pensado en aprendizajes integrados, experiencial y nuevas ecologías de aprendizaje puede proveer a las instituciones educativas un mejor entender de como aprenden sus actuales y futuros estudiantes y aprendices que habitaran esos espacios en busca de aprendizajes a lo largo de su vida, para atender a sus necesidades e inquietudes.

\section{Agradecimiento}

Esta publicación obtuvo el financiamiento de: El Fondo de Asistencia Internacional de los Estudiantes y Académicos Noruegos (SAIH).

\section{Lista de referencia}

Adúriz-Bravo, A. (2005). ¿Qué naturaleza de la ciencia hemos de saber los profesores deficiencias? Una cuestión actual de la investigación didáctica. Tecné, Episteme y Didaxis, 23-33.

Aguirre Pérez, C. y Vázquez Moliní, A. (2004). Consideraciones generales sobre la alfabetización científica en los museos de la ciencia como espacios educativos no formales. Revista electrónica de enseñanza de las ciencias, 3(3), 1-26.

Alves da Silva, C. y Ferreira, C. (2016). Las redes sociales y el aprendizaje informal de Estudiantes de Educación Superior. Acción Pedagógica, 25, 6-20.

Anderson, D., \& Ellenbogen, K. M. (2012). Learning science in informal contexts-Epistemological perspectives and paradigms. In Second international handbook of science education (pp. 1179-1187). Springer, Dordrecht.

Ausubel, D. (2002). Adquisición y retención del conocimiento. Una perspectiva cognitiva. Barcelona, España: Paidos

Bell, P., Lewenstein, B., W. Shouse, A. y A. Feder, M. (2009). Learning science in informal environments. People, places, and pursuits, $1^{\circ}$ edición. Washington: D.C., The National Academic Press.

Calero Vaquera, M. L. (2014). El discurso del WhatsApp: entre el Messenger y el SMS. Oralia, 17, 85-114.

Coll, C. (2013). La educación formal en la nueva ecología del aprendizaje: tendencias, retos y agenda de investigación. En RODRÍGUEZ ILLERA, J. L. (comp.) Educación y aprendizaje en la sociedad digital. Barcelona, Universitat de Barcelona, 156-170.

Coombs, P. H. y Ahmed, M. (1974). Attacking Rural Poverty: How Non formal Education Can Help. A Research Report for the World Bank Prepared by the International Council for Educational Development.

Corredor, M. (2003). Aula virtual: una alternativa de educación superior. Bucaramanga. Ediciones UIS.

Dewey, J. (1896). The reflex arc concept in psychology. Psychological Review 3: 357-370. Disponible en: http:// psychclassics.yorku.ca/Dewey/reflex.htm.

Donolo, D. (2019). ¡Tiene ideas de futuro y no es de mirar las estrellas! Identidad Profesional. En Paoloni, P., Rinaudo, M. C y Martín, R, Yo, tu... ellos y nosotros. Competencias socio-emocionales en la construcción de identidades profesionales. Córdoba: Editorial Brujas. (pp 209-248). 
Faure, P. (1972). informe "Aprender a ser: el nuevo mundo de la educación de hoy y de mañana" www.unesdoc. unesco.org/images/oo13/o01329/132984s.pdf

Fontanillas, T. R.; Catasús, M. G.; Raffaghelli, J. y Morer, A. S. (2020). Ecologías de aprendizaje para usar las TIC inspirándose en docentes referentes. Comunicar. Revista científica iberoamericana de comunicación y educación (62), 31-42.

Foresto E. (2020a). Aprendizajes formales, no formales e informales. Una revisión teórica holística. Contextos de Educación, 29 (21), 24-36. Recuperado de: http://www2.hum.unrc.edu.ar/ojs/index.php/ contextos/article/view/1142/1234

Foresto, E. (2020b, en prensa) ¿Qué es una leguminosa y cómo se clasifican? Una actualización para estudiantes de nivel medio y superior. Revista de Educación en Biología, 23(2).

Gasull, C. P., Herrador, E. S., \& Martín, R. B. (2020). Aprendizaje informal y prácticas agroecológicas en el contexto de una huerta. Revista de Ensino de Biologia da SBEnBio, 206-222.

Hager, P. (2012). Informal learning: everyday living. En The Routledge international handbook of learning. Oxon, Rouhagtledge, 207-215.

Huerta, R. y Alonso-Sanz, A. (Eds.). (2017). Entornos informales para educar en artes (Vol. 228). Universitat de València.

Ibáñez Etxeberria, A., Vicent Otaño, N., \& Asensio, M. (2012). Aprendizaje informal, patrimonio y dispositivos móviles: evaluación de una experiencia en educación secundaria. Didáctica de las ciencias experimentales y sociales.

Illera, J. L. R. (2018). EDUCACIÓN INFORMAL, VIDA COTIDIANA Y APRENDIZAJE TÁCITO. Teoría de la Educación; Revista Interuniversitaria, 30(1), 259-24.

Jackson, N. (2013). The concept of learning ecologies. En N. Jackson y B. Cooper (eds), Lifewide Learning, Education \& Personal Development (chapter A5). Recuperado de: http://www.lifewideebook.co.uk/ conceptual.html.

Lin, M., y Bound, H. (2011). To 'become'one: Developing professional identity through learning at work. In Proceedings of the 7 th international conference on researching work and learning (pp. 934-945).

Livingstone, D. W. (2006). Informal Learning: Conceptual Distinctions and Preliminary Findings. En BEKERMAN et al. (eds.) Learning in places. The Informal Education Reader. New York, Peter Lang, 203-228.

Martín, R. B. y Donolo, D. S. (2019). Aprendizajes informales. Perspectivas teóricas y relatos de aprendizajes. Ikastorratza, e-Revista de didáctica, (23)5. Recuperado de: https://dialnet.unirioja.es/servlet/ articulo? codigo $=7285765$

Matamala Riquelme, C. (2016). Uso de las TIC en el hogar: Entre el entretenimiento y el aprendizaje informal. Estudios pedagógicos (Valdivia) 42(3), 293-311.

Melgar, M. F., y Donolo, D. S. (2011). Salir del aula... Aprender de otros contextos: Patrimonio natural, museos e Internet. Revista Eureka sobre Enseñanza y Divulgación de las Ciencias, 8(3), 323-333.

Melnic, A. S. y Botez, N. (2014). Formal, non-formal and informal interdependence in education. Economy Transdisciplinarity Cognition 17(1), 113-119. 
Moreno, E. J. (2007). EL HERBARIO COMO RECURSO PARA EL APRENDIZAJE DE LA BOTÁNICA/The herbarium as a resource for the learning of Botany. Acta Botánica Venezuelica, 30(2), 415-427.

Moss, G. (2001). On Literacy and the Social Organisation of Knowledge Inside and Outside School. Language and Education, 15, 2-3, 146-161.

Rodríguez Illera, J. L. (2015). Los contextos y sus aprendizajes. Temps d’Educació, 48, 287-304.

Rodríguez Illera, J. L. y Kaechele, M. (2009). Digital Literacy and Activity Systems in Adolescents, en ZAJDA, J. y GIBBS, D. (eds.) Comparative Information Technology Languages, Societies and the Internet. New York, Springer Verlag, 89-102.

Salgado, E. Á., \& Guevara, H. A. (1998). El aprendizaje de algunos conceptos fundamentales en el campo de la botánica. Una experiencia investigación aula. Tecné Episteme y Didaxis: TED, (4).

Santos, I. E. M., \& Téllez, J. C. R. (2011). La identificación de organismos vegetales a partir del nombre común: un método útil para la enseñanza y el aprendizaje de la Botánica. Bio-grafía: escritos sobre la biología y su enseñanza, 4(7), 73-82.

Schugurensky, D. (2000). The forms of informal learning. Towards a conceptualization of the field. Working Paper 19-2000, Presentado en New Approaches for Lifelong Learning (NALL) Fourth Annual Conference, 6-8 de Octubre.

Trilla, J. (1986). La educación informal. Barcelona, PPU

Trilla, J. (1988). Animación Sociocultural, Educación y Educación no formal. Educar, 13, 17-41.

Trilla, J.; Gros, B.; López F. y Martín, M. J. (2003). La educación fuera de la escuela. Ámbitos no formales y Educación Social. Barcelona, España: Ariel Educación.

UNESCO (2015). Repensar laeducación ¿hacia un bien común universal? Declaración de Incheon. Educación, 2030.

Vadeboncoeur, J. (2006). Engaging Young People: Learning in Informal Contexts, en Green, J. y Luke, A. (eds.) Rethinking Learning: What Counts as Learning and What Learning Counts. Washington DC, AERA, 239-278.

Vicario, J. E., Chiecher, A. C., Amieva, R. L., Fernández, A., \& Ortiz, F. (2015). ¿Qué pasa Whatsapp? ¿Qué onda con la Física? 